\title{
Eigenkapitalnormen, Boni und Risikoanreize in Banken
}

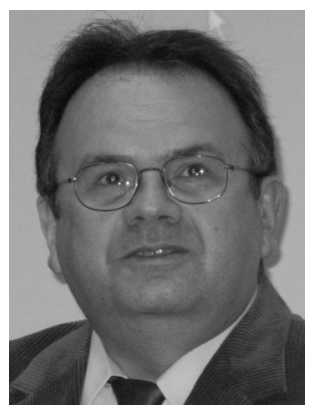

\section{Werner Neus}

Anreizsysteme, Banken, Regulierung, Risikoanreiz

Bonus Plans, Banks, Capital Regulation, Risk Incentive

Eine gängige Folklore zu Eigenkapitalnormen für Banken besagt, dass deren Verschärfung zur Milderung eines unerwünschten Risikoanreizes führt. Jedoch ist es in managergeleiteten Banken für das Aufkommen eines Risikoanreizes massgeblich, welche Anreize den Managern über deren Bonusprogramme vermittelt werden. Im Rahmen eines einfachen Modells wird gezeigt, dass die Zusammenhänge in mehrfacher Hinsicht uneindeutig sind. Zum einen kann je nach Ausgestaltung der Boni ein Risikoanreiz bestehen oder nicht. Zum anderen kann ein gegebenenfalls bestehender Risikoanreiz durch eine Verschärfung der Eigenkapitalnorm gemildert, verschärft oder gar nicht tangiert werden.

Generally, bank capital regulation is expected to mitigate the risk incentive problem. However, in focussing on the bank owners view conventional wisdom neglects the managers' decision making competence. Therefore, the managers' incentives induced by the bonus schemes they are faced with have to be taken into account. Using a simple model, it is shown that the relation between bonus schemes, capital regulation, and decisions on risk is ambiguous. First, there may or may not be a risk incentive. Second, given a risk incentive it may be mitigated, aggravated or not be influenced at all in case the capital regulation is tightened.

\section{Einführung}

Die gegenwärtig noch nicht überwundene Bankenkrise bringt es mit sich, dass sich die praktische Aufsicht und die Wissenschaft intensiv mit der Suche nach den Ursachen beschäftigen sowie damit, wie ähnlichen Fehlentwicklungen in der Zukunft vorgebeugt werden kann. Im Mittelpunkt steht die Abschätzung der Wirkungen regulativer Massnahmen mit dem Ziel, Risiken für einzelne Banken und für das gesamte Bankensystem zu verringern. Kern der Analyse sind in aller Regel die Anreizwirkungen, die von einer bestimmten Regulierung oder Nicht-Regulierung ausgehen.

Das in Banken aufgenommene Risiko wird durch die Erwartung beeinflusst, staatliche Instanzen könnten es sich buchstäblich nicht leisten, eine bestimmte Bank Pleite gehen zu lassen. Für diesen Fall ist zu vermuten, dass Banken hohe Risiken eingehen, weil im Fall des glücklichen Ausgangs damit ein höherer Erfolg einhergeht, während im Falle des unglücklichen Ausgangs der Staat die betreffende Bank auffängt. Daher geht es in den gegenwärtigen Diskussionen häufig um die Frage: Wie kann die tatsächliche Option gewährleistet werden, eine notleidende Bank abzuwickeln, ohne öffentliche Mittel heranzuziehen? 
Eng damit verbunden ist die Frage, ob es dafür erforderlich ist, das Universalbanksystem einzuschränken, weil es die Grösse, die Komplexität und die Unverzichtbarkeit einer bestimmten Bank sind, welche eine staatliche Rettung erforderlich zu machen scheinen.

Mehr, aber keineswegs ausschliesslich auf der Mikroebene setzt der Klassiker der bankaufsichtlichen Fragenstellungen an: Wie ist eine Eigenkapitalnorm auszugestalten, die einzelne Banken und das Bankensystem stabilisiert, ohne dabei die Kreditversorgung der Wirtschaft über Gebühr zu beeinträchtigen? Traditionell werden dem Bankeigenkapital die Funktionen der Risikoabdeckung - versinnbildlicht durch Begriffe wie „Verlustpuffer“ oder „Risikotragfähigkeit“ (Burghof/Rudolph 1996, 124 f.) - oder der Risikobegrenzung zugewiesen, wobei die zweite Sichtweise zu dominieren scheint (ebenda). Die damit verbundene Argumentation ist zunächst rein mechanisch. Dies wird besonders deutlich, wenn es heisst, für eine wirksame Risikobegrenzung komme es einzig darauf an, dass der risikolimitierende Faktor nicht beliebig vermehrbar sei (Schaber 2000, 59). Zwar ist dieser Gedanke grundsätzlich richtig, jedoch hat Eigenkapital auch eine verhaltensbeeinflussende Wirkung. Diese erkennt man in der ein funktionierendes marktwirtschaftliches System kennzeichnenden Haftung eines Entscheiders für die Folgen seiner Entscheidungen. Die Reichweite der risikobegrenzenden Anreizwirkung von Eigenkapital ist eine für die Bankenaufsicht zentrale Frage.

In Banken werden Entscheidungen in aller Regel nicht von den Eigentümern, sondern von angestellten Managern getroffen. Daher rücken statt oder wenigstens in Ergänzung zu den Anreizen der Eigentümer die Anreize der Manager in den Fokus des Interesses. Erhielte ein Manager ausschliesslich ein fixes Salär, wäre das Thema unter dem Gesichtspunkt der Risikobegrenzung unverdächtig. Da das Gehalt gezahlt wird, solange die Bank aktiv und der Manager unter Vertrag ist, hat dieser ein ausgeprägtes Interesse am Überleben der Bank sowie daran, dass sein Arbeitsverhältnis fortdauert. Durch Wahl eines geringen Risikos kann er dies am ehesten gewährleisten. Ein Fehlanreiz besteht allerdings möglicherweise in der Motivation, die Geschäftsstruktur der Bank so auszuweiten oder zu verkomplizieren, dass der Staat nicht umhin kann, in einer selbst bei geringem Risiko nicht ganz auszuschliessenden Bankenkrise die betreffende Bank zu retten. In jedem Fall wird die Sachlage komplizierter, wenn der Manager nicht lediglich ein Fixum, sondern auch einen erfolgsabhängigen, variablen Anspruch erhält, also einen Bonus.

Im vorliegenden Beitrag wird der gemeinsame Einfluss der Managerentlohnung und der geltenden Eigenkapitalnorm auf die Risikowahl in einer Bank analysiert. Der nachfolgende Überblick über die unmittelbar angrenzende Literatur beschränkt sich auf nach der Bankenkrise entstandene Beiträge, zeigt, dass das Themengebiet insgesamt alles andere als unerforscht ist, und identifiziert eine dennoch vorhandene Forschungslücke.

Einen Schwerpunkt im Schrifttum erkennt man in der empirischen Untersuchung des Zusammenhangs zwischen Managerentlohnung und Risiko der Banken. Nach Fablenbrach/Schulz (2011) wurde die Bankenkrise nicht dadurch ausgelöst, dass die Manager in eigenem Interesse und entgegen dem der Anteilseigner agierten. Demnach sei die Bankenkrise eher Folge bedauerlicher Irrtümer als falscher Anreize. Für die gegenteilige Folgerung plädieren Bebchuk/Cohen/Spamann (2010). Sie greifen die notleidend gewordenen Banken Bear Stearns sowie Lehman in Form von Fallstudien auf und analysieren detailliert das bisweilen vorgebrachte Argument, Manager hätten ja ihrerseits ebenfalls im Fall der Pleiten viel verloren und deshalb könne von einem Fehlanreiz ja keine Rede sein. Die Zahlen von Bebchuk et al. (2010) belegen eindrücklich, dass die betreffenden Topmanager in den 
der Krise vorangegangenen Jahren in Form von Cash-Boni und von veräusserten Aktien sowie Optionen ein Vielfaches der späteren Verluste vereinnahmt hatten. Bhagat/Bolton (2013) kommen in einer breiter angelegten Studie zu ähnlichen Ergebnissen. Vallascas/ Hagendorff (2012) untersuchen mit Daten aus den USA und aus Europa den Zusammenhang zwischen der Verwendung von Cash-Boni und dem Bankrisiko. Im Allgemeinen vermindern Cash-Boni das Ausfallrisiko, weil sie nur ausbezahlt werden, wenn die Bank solvent bleibt. Jedoch kehrt sich der Zusammenhang um, wenn die Insolvenzgefahr - gemessen durch die „distance to default“ nach Merton (1974) - zunimmt; der Zusammenhang kehrt sich auch dann um, wenn die Bank sich einem schwachen Aufsichtsregime gegenübersieht. Bai/Elyasiani (2013) führen ein Mass für die Risikosensitivität der Managerentlohnung ein („Vega“). Banken mit einem hohen Vega wiesen in der Bankenkrise eine geringere Stabilität auf. Erschwerend kommt hinzu, dass in grösseren Banken das Vega deutlich höher ist als in kleineren Banken. In einer modelltheoretischen Analyse beschäftigen sich Bannier/Feess/Packham (2013) mit der Frage, warum denn in einer Bank Bonus-Modelle eingesetzt werden, die schliesslich ein so grosses Risiko herbeiführen. Sie identifizieren dabei zwei einander verstärkende Wirkungskanäle: Ein hoher Anteil an erfolgsabhängiger Entlohnung ist ein Instrument zur Akquisition besonders talentierter Mitarbeiter, für die eine erfolgsabhängige Entlohnung relativ attraktiver ist als für untalentierte Mitarbeiter. Dieser Effekt wird verstärkt durch den Wettbewerb um die talentierten Mitarbeiter. Auch Maug/Albrecht (2011) sowie Gabaix/Landier (2008) führen die Dynamik der Managergehälter auf den Wettbewerb um das knappe Führungstalent zurück.

Als weiteren Schwerpunkt kann man die Interaktion der Problemfelder Entlohnung sowie staatliche Aktivitäten bei der Risikowahl der Bank identifizieren. Nach Chaigneau (2013) führen potentielle öffentliche Garantien ebenso wie Einlagenversicherungen dazu, dass die externen Effekte eines hohen Risikos nicht vollständig internalisiert werden. Daher gestalten Bankeigentümer die Anreizverträge mit Bankmanagern so, dass diese ein exzessives Risiko wählen. Aus diesem Grund plädiert Chaigneau für eine geeignet ausgestaltete Vergütungsregulierung sowie für Sanktionen für Manager im Fall eines Defaults. Ähnlich analysieren Hakenes/Schnabel (2013) den Zusammenhang zwischen der Erwartung eines Bail-outs und der Ausgestaltung von Anreizschemata. Auf die Erwartung eines Bailouts reagieren die Eigentümer, indem sie ein besonders konvexes Bonusschema wählen, so dass der Manager ein besonders hohes Risiko wählt. Bei einer starken Zunahme der Bailout-Erwartungen sollte die regulierende Instanz Obergrenzen für Boni einführen, um dem induzierten Risikoanreiz Einhalt zu gebieten. Anders als bei Chaigneau (2013) erweist sich dagegen eine Verschärfung der Managerhaftung als kontraproduktiv, weil auch der Manager von dem Bail-out profitiert. Vor dem Hintergrund des Bankenwettbewerbs arbeiten Hakenes/Schnabel (2010) theoretisch heraus, dass die Erwartung des Bail-outs bei einer Bank zugleich deren Wettbewerb mit anderen Banken verzerrt: Die Neigung der vielleicht zu rettenden Bank, eine expansive Strategie zu wählen, bringt es mit sich, dass konkurrierende Banken ihr Risiko erhöhen. Bhagat/Bolton/Lu (2012) heben die Grösse von Finanzunternehmen als Proxy für „,too big to fail“ und also für die Wahrscheinlichkeit eines Bailouts hervor. Tatsächlich gehen grössere Institute ein höheres Risiko ein, und dies vor allem durch einen höheren Verschuldungsgrad. Eine hohe Aktienbeteiligung des Vorstandsvorsitzenden (gemessen am Median-Vorstand) ist dagegen mit einer Verringerung des Risikos verbunden. 
Die zuletzt angeführte Gruppe von Beiträgen lässt bereits erkennen, dass es einen Einfluss auf die Ergebnisse hat, wenn man nicht lediglich eine Determinante für das Bankrisiko in den Mittelpunkt rückt, sondern mehrere. Eine solche Untersuchung liefert die empirische Studie von Laeven/Levine (2009). Bei ihnen ist die Eigentümerstruktur eine mediierende Variable, welche die Auswirkung des Eigenkapitals auf die Risikowahl steuert. Während in Banken mit ausgeprägtem Streubesitz das Eigenkapital keinen deutlichen Einfluss auf das Risiko ausübt, führen schärfere Eigenkapitalnormen in Banken mit einem starken Eigentümer zu einer Risikoerhöhung - was angesichts der geringeren Diversifikation durchaus als Überraschung angesehen werden darf.

Das letztgenannte Manuskript weist insofern eine deutliche Parallele zur vorliegenden Untersuchung auf, als dort wie hier der Einfluss einer mediierenden Variablen auf den $\mathrm{Zu}$ sammenhang zwischen Eigenkapitalnorm und Risikowahl untersucht wird. Die vorgelagerte Frage ist hier die nach dem Einfluss der Managerentlohnung auf das Bankrisiko. Im Mittelpunkt stehen nicht die Höhe der Boni oder deren zeitliche Verteilung, sondern ihre Bemessungsgrundlage. Führt das Zusammenspiel von Bemessungsgrundlage und Entlohnungsfunktion zu einem im Cash-flow konvexen Anspruch des Managers, kommt es zum Risikoanreiz. Die zentrale Frage ist sodann, ob und wie dieser Zusammenhang durch eine Verschärfung der Eigenkapitalnorm beeinflusst wird, wie sie die Umsetzung von Basel III durch das Anfang 2014 in Kraft getretene CRD IV-Paket vorsieht. ${ }^{1}$ Es stellt sich heraus, dass in Abhängigkeit von der Bemessungsgrundlage für die Boni die Eigenkapitalnorm einen vorhandenen Risikoanreiz mildert, verstärkt oder gar nicht beeinflusst. Demnach sollte die Eigenkapitalnorm stets in ein Geflecht anderer Regelungen eingebunden sein, damit die intendierte Wirkung nicht konterkariert wird.

Die weiteren Ausführungen sind wie folgt gegliedert. Im zweiten Abschnitt wird ein möglichst einfach gehaltenes Modell präsentiert, das es zulässt, den Zusammenhang zwischen Bonus, Eigenkapitalnorm und Risikowahl in leicht nachvollziehbarer Weise zu untersuchen. Eine Risikoerhöhung wird durch den Mean-Preserving Spread im Sinne von Rothschild/Stiglitz (1970) abgebildet. Im Anschluss an die ausführliche Analyse des Grundmodells werden in Abschnitt 3 zur Überprüfung der Robustheit der Ergebnisse zwei Varianten aufgegriffen: zum einen die Verringerung des Risikos im Sinne der Ausfallwahrscheinlichkeit, was dann auch eine Veränderung des erwarteten Cash-flows mit sich bringt, zum anderen die Verallgemeinerung der Annahme über die Cash-flow-Verteilung. In der Hauptsache erweisen sich die Ergebnisse des Basismodells als robust. Im abschliessenden vierten Abschnitt werden Ansatz und Ergebnisse kritisch diskutiert.

\section{Bonus, Eigenkapitalnorm und Risikowahl}

\subsection{Das Modell}

Gegenstand der folgenden Ausführungen ist ein extrem sparsam parametrisiertes Modell zur Illustration der Interaktion von Managerentlohnungssystemen und Eigenkapitalnormen bei den Risikoentscheidungen einer Bank. Im Kern handelt es sich um eine vereinfachte Variante des Modellrahmens von Hakenes/Schnabel (2013).

1 Das CRD IV-Paket besteht aus der Richtlinie Capital Requirements Directive in der vierten Auflage sowie aus der Verordnung Capital Requirements Regulation; siehe dazu übersichtlich Luz et al. (2013). 
Das Geschäft der von einem risikoneutralen Manager geleiteten Bank erfordert einen Gesamtbedarf an Refinanzierung in Höhe von 1; davon wird ein Anteil $e$ in Form von Eigenmitteln aufgebracht, der komplementäre Anteil $d=1-e$ besteht aus Depositen oder anderen Formen von Fremdkapital. Die somit verfügbaren Mittel werden für eine Geschäftsstruktur verwendet, die - ohne dass dies näher spezifiziert wird - das Kreditgeschäft und den Eigenhandel umfasst. Aus dem Gesamtgeschäft resultiert am Ende des einperiodigen Zeithorizontes ein Brutto-Cash-flow $y$ vor Kapitalkosten und Managerbonus mit der Eigenschaft

$$
y=\left\{\begin{array}{ccc}
0 & \text { mit } & p_{0}=\frac{1}{3}+\hat{\rho} \\
x & \text { mit } & p_{1}=\frac{1}{3}-2 \hat{\rho} . \\
2 x & \text { mit } & p_{2}=\frac{1}{3}+\hat{\rho}
\end{array}\right.
$$

Eine solche Dreipunktverteilung ist die schlichteste Form, die es zulässt, Formen der Managerentlohnung einzubeziehen, die im Ergebnis $y$ konvexe oder konkave Ansprüche darstellen. Der Handlungsparameter des Managers $\hat{\rho}$ nimmt keinen Einfluss auf den erwarteten Cash-flow $E\{y\}=x$, sondern gestaltet allein das Risiko. Die Wahl eines höheren $\hat{\rho}$ bewirkt einen Mean-Preserving Spread und führt zu einer im Sinne der stochastischen Dominanz zweiten Grades inferioren Verteilung des Cash-flows. Für das Weitere wird unterstellt, dass der Manager entscheiden kann, ob der Risikoparameter den Wert $\hat{\rho}=0$ oder $\hat{\rho}=\rho$ (mit $0<\rho<1 / 6)$ annimmt. Die Implementierung des höheren Risikos ist für den Manager mit Kosten $c$ verbunden, die er in Kauf nehmen muss, um gegenüber der Bankenaufsicht die unerwünschte Risikoerhöhung zu verschleiern. Es gilt also

$$
c(\hat{\rho})= \begin{cases}c & \text { wenn } \hat{\rho}=\rho \\ 0 & \text { wenn } \hat{\rho}=0\end{cases}
$$

Angesichts des konstanten Erwartungswertes ist eine mit Kosten verbundene Risikoerhöhung nach Massgabe des erwarteten Wertes aller Ansprüche strikt suboptimal; die firstbest-optimale kritische Kosten-Schranke für die Erhöhung des Risikos beträgt also $c^{*}=0$. Als Kriterium für das Vorliegen und die Stärke eines Risikoanreizes wird nachfolgend untersucht, wie hoch die kritische Kostenschranke der Risikoerhöhung aus des Sicht des Managers ist. Ist die Kostenschranke des Managers positiv, liegt ein schädlicher Risikoanreiz vor.

Aus dem Cash-flow müssen die Kapitalkosten bestritten werden. Der Fremdkapitalkostensatz beträgt $r_{d}$ und setzt sich zusammen aus Tilgung, Einlagenzins und einer Prämie für die Einlagenversicherung. Für den Eigenkapitalkostensatz gilt $r_{e}>r_{d}$, was sich beispielsweise mit steuerlichen Wirkungen begründen lässt. Die erwartete Rentabilität des Portefeuilles ist gross genug, um unabhängig von der Finanzierung als lohnend zu gelten; dies impliziert $x>r_{e}$. Die Bedingung impliziert zugleich, dass die Insolvenzwahrscheinlichkeit stets $p_{0}=1 / 3+\hat{\rho}$ beträgt. Sie wird also ausschliesslich durch die Risikowahl determiniert und nicht unmittelbar durch die Eigenkapitalausstattung beeinflusst, wie es bei einer kontinuierlichen Verteilung der Fall wäre. 
In den drei folgenden Abschnitten wird für verschiedene Bonus-Modelle untersucht, ob und wie stark sich daraus ein Risikoanreiz ergibt sowie ob Vorliegen und Stärke des Risikoanreizes durch die Ausgestaltung der Eigenkapitalnorm beeinflusst werden. Für die Parameter der Bonus-Modelle gilt die generelle Beschränkung, dass der Bonus kleiner ist als der realisierte Brutto-Cash-flow.

\subsection{Konstanter Bonus oberhalb einer gewissen Gewinnschwelle}

Das erste untersuchte Bonus-Modell sieht zusätzlich zu einem im Brutto-Cash-flow bereits enthaltenen, erfolgsunabhängigen Basisgehalt einen konstanten Bonus $b$ vor, der ausbezahlt wird, wenn eine kritische Gewinnschwelle erreicht wird. In einem Dreizustandsmodell ist danach zu unterscheiden, ob die kritische Gewinnschwelle oberhalb des mittleren Cash-flows $x$ liegt oder nicht.

Das Bonus-Modell 1 sieht vor, dass der Bonus ausgezahlt wird, wenn die Eigentümer einen positiven Gewinn nach Eigenkapitalkosten erzielen. Gegeben die übrigen Annahmen bedeutet dies, dass lediglich bei völligem Fehlschlag des Projekts kein Bonus ausgezahlt wird. Für den erwarteten Gewinn des Managers gilt in diesem Fall

$\mathrm{E}\left\{\pi_{1}\right\}=\left(p_{1}+p_{2}\right) b_{1}-c(\hat{\rho})=\left(\frac{2}{3}-\hat{\rho}\right) b_{1}-c(\hat{\rho})$.

Wie unmittelbar ersichtlich ist, mindert eine vermeidbare Risikoerhöhung den erwarteten Gewinn des Managers, weil der erwartete Bonus sinkt und überdies Kosten anfallen. Daher wählt der Manager stets das minimale Risiko. Die ökonomische Logik ist leicht ersichtlich: Die Anspruchsstruktur des Managers ist wegen des nur in der Insolvenz wegfallenden Bonus konkav (vgl. Abbildung 1) und provoziert damit risikoarme Entscheidungen.
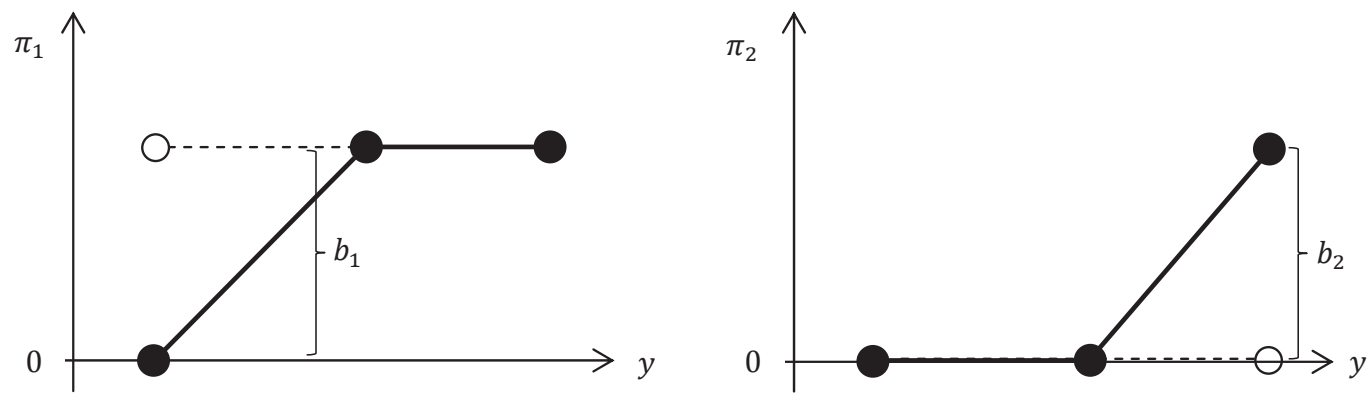

Abbildung 1: Manageranspruch bei fixem Bonus und niedriger bzw. hoher Gewinnschwelle

In den Abbildungen 1 bis 3 sind die Ansprüche des Managers durch die fetten Punkte gekennzeichnet. Die der Sache nach unmassgeblichen durchgezogenen Linien verdeutlichen die Konvexität oder Konkavität der Ansprüche, die gestrichelten Linien zeigen jeweils einen durchgängig linearen Verlauf des Bonus an, der einen in Bezug auf das Risiko neutralen Anreiz hervorriefe. 
Beim Entlohnungsmodell 2 ist die Gewinnschwelle so hoch angesetzt, dass der Bonus nur im Falle eines überdurchschnittlich hohen Cash-flows ausgezahlt wird. Der erwartete Gewinn des Managers beträgt dann

$\mathrm{E}\left\{\pi_{2}\right\}=p_{2} b_{2}-c(\hat{\rho})=\left(\frac{1}{3}+\hat{\rho}\right) b_{2}-c(\hat{\rho})$.

Nun besteht ein Trade-off zwischen der Höhe des erwarteten Bonus und den Kosten der Risikoerhöhung. Das erhöhte Risiko ist für den Manager von Vorteil, wenn gilt

$c<c_{2} \equiv \rho b_{2}$.

Anders als bei niedriger Gewinnschwelle wählt der Manager also das höhere Risiko, wenn die Kosten für die Risikoerhöhung nicht zu hoch sind. Weiter liegt die kritische Kostenschranke um so höher, je höher der Bonus ist. Die rechte Graphik in Abbildung 1 zeigt deutlich auf, dass das Krümmungsverhalten des Entlohnungsschemas verantwortlich für das Ergebnis ist: Konvexe Ansprüche befördern den Risikoanreiz.

Für beide Ausgestaltungsformen eines konstanten Bonus gilt, dass die Eigenkapitalnorm keinen Einfluss auf die Risikowahl ausübt.

\subsection{Variabler Bonus auf Basis des bilanziellen Gewinns}

Das zweite Bonus-Modell besteht in einer Beteiligung in Höhe eines Anteils $\beta$ am bilanziell gemessenen Gewinn, der sich bei den vorliegenden Bedingungen ergibt als der Cashflow abzüglich der Fremdkapitalkosten. Da der Abzug der Fremdkapitalkosten nur bei einem positiven Cash-flow zur Geltung kommt, resultiert eine konvexe Anspruchsstruktur (Abbildung 2).

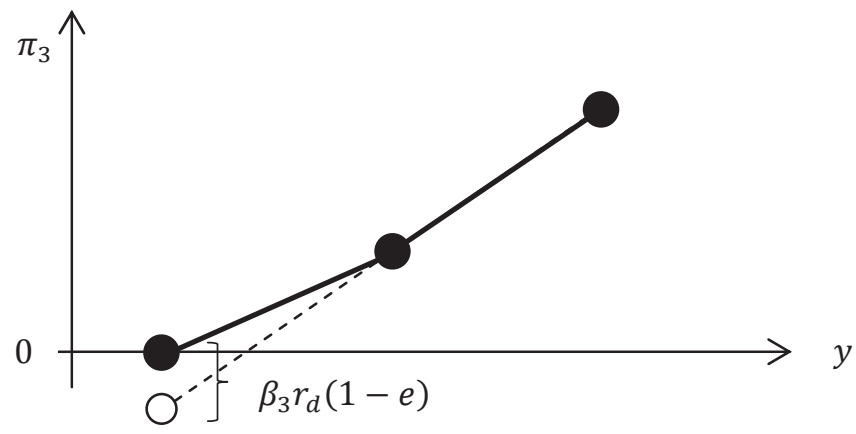

Abbildung 2: Konvexer Manageranspruch bei Bonus auf Basis des bilanziellen Gewinns

Man erhält für den erwarteten Gewinn des Managers

$$
\begin{aligned}
\mathrm{E}\left\{\pi_{3}\right\} & =p_{1} \beta_{3}\left(x-r_{d}(1-e)\right)+p_{2} \beta_{3}\left(2 x-r_{d}(1-e)\right)-c(\hat{\rho}) \\
& =\beta_{3}\left(x-\left(\frac{2}{3}-\hat{\rho}\right) r_{d}(1-e)\right)-c(\hat{\rho}) .
\end{aligned}
$$

In Anbetracht seiner Ansprüche erweist sich für den Manager die Wahl des hohen Risikos als vorteilhaft, wenn gilt 
$c<c_{3} \equiv \beta_{3} \rho r_{d}(1-e)$.

Aus der konvexen Form der Ansprüche des Managers ergibt sich naturgemäss ein Risikoanreiz. Hervorzuheben ist nun aber, dass die Stärke des Risikoanreizes von der Eigenkapitalquote abhängt. Konkret nimmt die kritische Schranke für die Kosten der Risikoerhöhung einen geringeren Wert an, wenn mehr Eigenkapital zur Finanzierung herangezogen wird. Darin schlägt sich die intendierte Wirkung einer Eigenkapitalnorm nieder, nämlich über eine verschärfte Beteiligung an den Verlustgefahren externe Effekte zu internalisieren und somit den Risikoanreiz zu verringern. Die Gleichungen und die Abbildung 3 weisen es unmittelbar als „Vorteil“ der Verschuldung aus, dass sie im Insolvenzfall nicht bedient wird. Dieser Vorteil verringert sich durch die reine Bilanzmechanik, wenn ein grösserer Teil des Fremdkapitals infolge einer verschärften Eigenkapitalnorm durch Eigenkapital ersetzt wird.

\subsection{Variabler Bonus auf Basis des Residualgewinns}

Als letzte Variante wird nunmehr ein Bonus auf Basis des Residualgewinns in Betracht gezogen. Der Residualgewinn (oder Economic Value Added) unterscheidet sich vom bilanziellen Gewinn dadurch, dass auch die Eigenkapitalkosten bei der Bestimmung des Überschusses abgezogen werden. Dieser zusätzliche Abzug hat aus Sicht des Managers die Auswirkung, dass in den erfolgreichen Zuständen eine geringere Anspruchsgrundlage verbleibt, mit der Implikation, dass der Insolvenzzustand relativ attraktiver wird, weil mit der Zahlungseinstellung eine „Entlastung“ um diesen höheren Betrag verbunden ist. All dies erkennt man gleichermassen aus der analytischen Darstellung wie aus Abbildung 3.

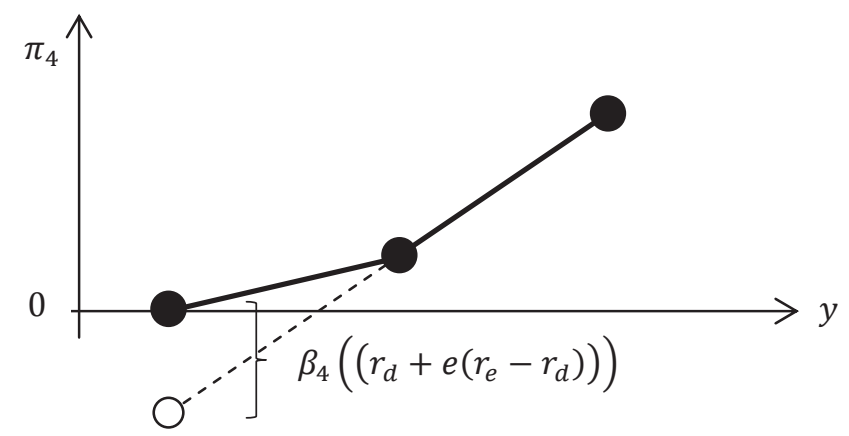

Abbildung 3: Konvexer Manageranspruch bei Bonus auf Basis des Residualgewinns

Für den erwarteten Gewinn des Managers gilt im Falle dieses vierten Bonus-Modells

$$
\begin{aligned}
\mathrm{E}\left\{\pi_{4}\right\} & =p_{1} \beta_{4}\left(x-\left(r_{d}+e\left(r_{e}-r_{d}\right)\right)\right)+p_{2} \beta_{4}\left(2 x-\left(r_{d}+e\left(r_{e}-r_{d}\right)\right)\right)-c(\hat{\rho}) \\
& =\beta_{4}\left(x-\left(\frac{2}{3}-\hat{\rho}\right)\left(r_{d}+e\left(r_{e}-r_{d}\right)\right)\right)-c(\hat{\rho}) .
\end{aligned}
$$

Für die Entscheidung des Managers über das höhere Risiko ergibt sich somit das folgende Kriterium:

$c<c_{4} \equiv \beta_{4} \rho\left(r_{d}+e\left(r_{e}-r_{d}\right)\right)$. 
Auch hier ist der Manager wegen seiner konvexen Ansprüche einem Risikoanreiz ausgesetzt. Jedoch zeigt sich ein bedeutender Unterschied in Bezug auf die Wirkung der Eigenkapitalnorm: Massgeblich für das Ausmass der Konvexität sind nunmehr die gesamten Kapitalkosten. Sofern diese bei einer Eigenkapitalausweitung steigen, wählt der Manager ein um so grösseres Risiko, je schärfer die Eigenkapitalnorm ist. Im Falle einer Managerentlohnung auf Basis des Residualgewinns muss also überprüft werden, wie sich die durchschnittlichen Kapitalkosten entwickeln, wenn mehr Eigenkapital eingesetzt wird. Wenn Eigenkapitalnormen überhaupt eine Bindungswirkung entfalten, führen sie zu einer Verteuerung der Gesamtfinanzierung. ${ }^{2}$ Vorliegend ist also eine Risikoerhöhung im Falle von schärferen Eigenkapitalnormen zu erwarten. Es zeigt sich demnach, dass eine zunächst unverfänglich erscheinende und der Sache nach gewiss nicht unplausible Modifikation der Bemessungsgrundlage für einen Bonus - in einer Art, wie sie von Murphy/Jensen (2011, 32 ff.) ausdrücklich empfohlen wird - zu einer Umkehrung der komparativen Statik in Bezug auf die Wirkung einer Eigenkapitalnorm führt. Vermeintlich gesicherte Erkenntnisse sind insofern zu relativieren.

\subsection{Synopse und Ergebnis}

Die untersuchten Bonus-Modelle lassen sich wie gesehen im Hinblick auf die Wirkung der Eigenkapitalnorm analysieren. Ohne weiteres ist es aber nicht möglich, sie untereinander zu vergleichen. Zu diesem Zweck wird nun eine Normierung der Boni vorgenommen. Die jeweiligen Bonusparameter $b_{1}, b_{2}, \beta_{3}$ und $\beta_{4}$ werden so festgelegt, dass der erwartete Bonus im Fall des geringen Risikos übereinstimmt. Die sich dann einstellenden Ergebnisse sind in Tabelle 1 zusammengefasst:

\begin{tabular}{cccc} 
Modell & $\begin{array}{c}\text { erwarteter Bonus } \\
\text { bei niedrigem Risiko }\end{array}$ & Entlohnungsparameter & $\begin{array}{c}\text { kritische Schranke } \\
\text { für die Kosten }\end{array}$ \\
\hline 1 & $B=\frac{2}{3} b_{1}$ & $b_{1}=\frac{3}{2} B$ & $c_{1}=-\rho B \cdot \frac{3}{2}<0$ \\
2 & $B=\frac{1}{3} b_{2}$ & $b_{2}=3 B$ & $c_{2}=\rho B \cdot 3$ \\
3 & $B=\beta_{3}\left(x-\frac{2}{3} r_{d}(1-e)\right)$ & $\beta_{3}=\frac{B}{x-\frac{2}{3} r_{d}(1-e)}$ & $c_{3}=\rho B \cdot \frac{r_{d}(1-e)}{x-\frac{2}{3} r_{d}(1-e)}$ \\
4 & $B=\beta_{4}\left(x-\frac{2}{3}\left(r_{d}+e\left(r_{e}-r_{d}\right)\right)\right)$ & $\beta_{4}=\frac{B}{x-\frac{2}{3}\left(r_{d}+e\left(r_{e}-r_{d}\right)\right)}$ & $c_{4}=\rho B \cdot \frac{r_{d}+e\left(r_{e}-r_{d}\right)}{x-\frac{2}{3}\left(r_{d}+e\left(r_{e}-r_{d}\right)\right)}$ \\
\hline
\end{tabular}

Tabelle 1: Synopse zu den vier Bonus-Modellen

Der Vergleich der kritischen Schranken zeigt, dass $c_{1}<c_{3}<c_{4}<c_{2}$. Die Relationen sind gut nachvollziehbar, weil bei einem konstanten Bonus, der nur im günstigsten Fall ausbezahlt wird, die Krümmung der Bonusfunktion am stärksten ist, was mit dem intensivsten Risikoanreiz einhergeht. Die dafür erforderlichen Randbedingungen kann man

2 Vor dem Hintergrund der Argumentation von Admati et al. (2011) sollte hervorgehoben werden, dass dies keineswegs impliziert, hohe Eigenkapitalanforderungen seien für ein funktionierendes Bankgeschäft unzumutbar teuer. 
kaum als besonders kritisch ansehen. Gefordert wurde lediglich, dass das Geschäftsmodell der Bank unabhängig von der Finanzierung im Durchschnitt rentabel ist $\left(x>r_{e}\right)$ und dass Eigenkapital grundsätzlich teurer ist als Fremdkapital $\left(r_{e}>r_{d}\right)$, so dass die Eigenkapitalnorm bindet.

Aus der allgemeinen Finanztheorie ist wohlbekannt, dass sich Risikoanreize entlang des Kriteriums der Konkavität oder Konvexität der Zielgrösse erklären lassen. Sofern die Entlohnung zu konvexen Ansprüchen führt (vorliegend also bei den Modellen 2, 3 und 4), kann die Auswirkung einer Eigenkapitalnorm danach beurteilt werden, ob sie die Konvexität der Manageransprüche verschärft oder abschwächt. Die untersuchten Modelle zeigen, dass grundsätzlich alle Vorzeichen möglich sind: kein Einfluss, verringerter Risikoanreiz und verschärfter Risikoanreiz. ${ }^{3}$

Die Einbeziehung normierter durchschnittlicher Boni zeigt überdies, dass ein höheres Niveau der Boni zwar bestehende Risikoanreize verschärft. Insofern ist die in der CRD IV verlangte Limitierung der Boni relativ zum Fixgehalt keineswegs zu kritisieren. Jedoch ist zu konstatieren, dass die Höhe eines Bonus keine Rolle spielt für die Interaktion mit der Eigenkapitalnorm. Dies lässt die Folgerung zu, dass die Bankenaufsicht auch den Bemessungsgrundlagen für einen Bonus die gebotene Aufmerksamkeit widmen sollte.

\section{Zur Robustheit des einfachen Modells}

Angesichts der extrem einfachen Modellierung scheint es angebracht, zur Überprüfung der Robustheit der Ergebnisse Varianten des vorgestellten Basisansatzes heranzuziehen. Dies geschieht nachfolgend in zweierlei Hinsicht. Zum einen wird als Aktionsparameter des Managers statt der reinen Risikoerhöhung eine Verringerung der Ausfallwahrscheinlichkeit einbezogen; zum anderen wird der Fall eines im Bereich positiver Werte kontinuierlich verteilten Cash-flows analysiert. Um die analytischen Aspekte nicht zu sehr in den Mittelpunkt zu rücken, wird jeweils der Ansatz eingeführt, um anschliessend gleich zu den Ergebnissen und Interpretationen überzuleiten. ${ }^{4}$

\subsection{Bonus, Eigenkapitalnorm und Kreditüberwachung}

Die Risikowahl des Managers wurde in Abschnitt 2 als Gestaltung des reinen Risikos im Sinne des Mean-Preserving Spread abgebildet. Für den Eigenhandel ist diese Modellierung adäquat. Im Kreditgeschäft einer Bank wäre es hingegen ein eher ungewöhnlicher Eingriff in die Risikostruktur des Kreditportefeuilles. Dagegen gehört es zu den kennzeichnenden Aufgaben eines Managers im Kreditgeschäft, durch eine intensive Kreditwürdigkeitsprüfung vor der Kreditvergabe und durch eine intensive Kreditüberwachung nach Kreditvergabe die Ausfallwahrscheinlichkeit zu verringern; um die Ausführungen sprachlich nicht zu überladen, wird anschliessend durchweg auf die Kreditüberwachung Bezug genommen. Diese wird nun als Effortanreizproblem abgebildet. Konkret soll für den Brutto-Cash-flow $y$ der Bank gelten

3 Massgeblich für diese Folgerung sind nicht die normiert ausgewiesenen kritischen Schwellen aus Tabelle 1, sondern die in den Abschnitten 2.2-2.4 genannten Schwellenwerte. Die komparative Statik ist darin offensichtlich.

4 Ausführlichere Herleitungen sind auf Anfrage beim Verfasser erhältlich. 


$$
y=\left\{\begin{array}{lll}
0 & \text { mit } & p_{0}=\frac{1}{3}-2 \hat{\theta} \\
x & \text { mit } & p_{1}=\frac{1}{3}+\hat{\theta} \\
2 x & \text { mit } & p_{2}=\frac{1}{3}+\hat{\theta}
\end{array}\right.
$$

Der Aktionsparameter $\hat{\theta}$ steht dabei für die Intensität der Kreditüberwachung und bringt im Falle einer hohen Ausprägung $\theta$ (mit $0<\theta<1 / 6$ ) eine verringerte Ausfallwahrscheinlichkeit sowie einen erhöhten Erwartungswert des Brutto-Cash-flows mit sich. Zugleich sind damit für den Manager persönliche Kosten in Höhe von $k$ verbunden:

$k(\hat{\theta})=\left\{\begin{array}{ll}k & \text { wenn } \hat{\theta}=\theta \\ 0 & \text { wenn } \hat{\theta}=0\end{array}\right.$.

Die durch eine intensive Kreditüberwachung bewirkte, kostenträchtige Verringerung der Ausfallwahrscheinlichkeit ist nach Massgabe des erwarteten Wertes aller Ansprüche lohnend, wenn die damit verbundene Erhöhung des erwarteten Cash-flows hinreichend gross ist, um die privaten Kosten des Managers zu kompensieren. Die dafür erforderliche Relation $k<k^{*} \equiv 3 \theta x$ gibt eine Benchmark für die Beurteilung der Managerentscheidungen ab. Sind die aus Sicht des Managers relevanten kritischen Schranken für die Kosten niedriger als $k^{*}$, kommt es zu einem Fehlanreiz in Form der Unterinvestition in die Kreditüberwachung; zugleich ist damit ein zu hohes Ausfallrisiko verbunden. Für das Weitere wird unterstellt, dass die tatsächlichen Kosten nicht grösser sind als $k^{*}$, so dass eine intensive Kreditüberwachung sozial optimal ist.

Auf dieser Basis lassen sich nun die vier oben eingeführten Bonus-Modelle analysieren und in ihren Auswirkungen wie folgt zusammenfassen:

1. In allen Modellen kommt es zu einer Unterinvestition in die Kreditüberwachung.

2. Bei Normierung des erwarteten Bonus nehmen die kritischen Kostenschranken stets den gleichen Wert an. Die Stärke des Unterinvestitionsproblems hängt also nicht von der Ausgestaltung des Bonus-Modells ab. Das Niveau des Bonus hat hier allerdings durchgängig einen positiven Einfluss: Je höher der erwartete Bonus, desto geringer ist das Unterinvestitionsproblem.

3. Bei den Modellen mit konstantem Bonus oberhalb einer kritischen Gewinnschwelle hat die Eigenkapitalnorm keinen Einfluss auf den kritischen Schwellenwert für die Kosten.

4. Bei einem zum bilanziellen Gewinn proportionalen Bonus erhöht eine Verschärfung der Eigenkapitalnorm den kritischen Schwellenwert für die Kosten und verringert somit das Unterinvestitionsproblem.

5. Dagegen zeigt sich im Falle des zum Residualgewinn proportionalen Bonus wie in Abschnitt 2 die gegenteilige Wirkung: Eine schärfere Eigenkapitalnorm geht mit einem niedrigeren Schwellenwert und somit mit einer stärkeren Unterinvestition einher, wenn die Eigenkapitalnorm bindet.

In Bezug auf die zentrale Fragestellung des Beitrags, nämlich die Interaktion zwischen der Eigenkapitalnorm, der Ausgestaltung von Bonus-Modellen und drohenden Fehlanreizen zeigen sich also die gleichen Wirkungen wie im Falle des reinen Risikoanreizes. 


\subsection{Risikowahl und kontinuierlich verteilter Cash-flow}

Die bislang verwendete Annahme eines diskret verteilten Cash-flows lässt sich nur mit Vereinfachungsgründen erklären. Die konkret unterstellte Verteilung hat zudem die Implikation, dass die Eigenkapitalausstattung keinen unmittelbaren Einfluss auf die Insolvenzwahrscheinlichkeit hat. Nun ist zu überprüfen, ob die Vereinfachung eine kritische Voraussetzung für die genannten Ergebnisse ist. Dafür wird unterstellt, dass der Cash-flow der Bank im Intervall nichtnegativer Cash-flows kontinuierlich verteilt ist: $y \sim(0, \infty) . f(\cdot)$, $F(\cdot)$ und $\varphi(\cdot)$ kennzeichnen die zweimal stetig integrierbare Dichtefunktion, die Verteilungsfunktion bzw. das Integral über die Verteilungsfunktion des Cash-flows sowie $\mu$ für dessen konstanten Erwartungswert.

Besondere Aufmerksamkeit verdient die $\varphi$-Funktion, die es ermöglicht, in übersichtlicher Form die reine Risikoerhöhung in die Analyse einzubeziehen. Der Mean-Preserving Spread ist äquivalent zu einer Verschlechterung nach Massgabe der stochastischen Dominanz zweiten Grades bei konstantem Erwartungswert (Rothschild/Stiglitz 1970). Von zwei Verteilungen $G$ und $H$ ist $G$ genau dann stochastisch dominant zweiten Grades, wenn für alle Elemente $y$ des Wertebereichs der Zufallsvariablen $\varphi_{G}(y) \leq \varphi_{H}(y)$ sowie für mindestens ein $y$ die strenge Ungleichung gilt (Neus 2013, 509). Daher steht die $\varphi$-Funktion unmittelbar und ausschliesslich für die reine Risikowirkung. Je grösser der Funktionswert bei konstantem Argument, desto höher ist das Risiko.

Einige der Zwischenergebnisse hängen von der Gestalt der Verteilungen des Cash-flows ab. Massgeblich ist, wie häufig sich die Verteilungsfunktionen vor und nach Risikoerhöhung schneiden. Die nachstehenden Abbildungen zeigen, dass bei einem Mean-Preserving Spread verschiedene Fälle denkbar sind. Abbildung 4 zeigt den Fall nur eines Schnittpunktes, wie er sich beispielsweise in der Klasse symmetrischer unimodaler Verteilungen ergibt.

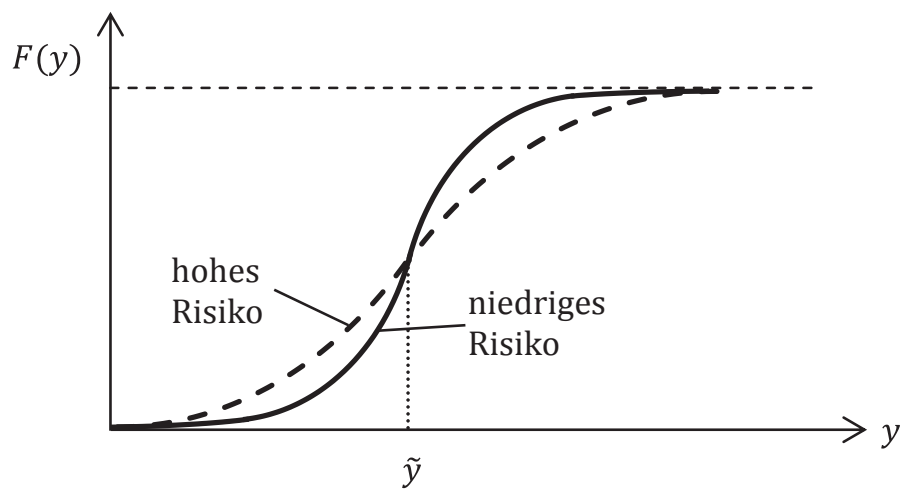

Abbildung 4: Verteilungsfunktionen mit nur einem Schnittpunkt

Abbildung 5 zeigt ein Beispiel für symmetrische bimodale Verteilungen mit mehreren Schnittpunkten. Wie in Abbildung 4 steht die Funktion mit dem gestrichelten Verlauf für das höhere Risiko. 


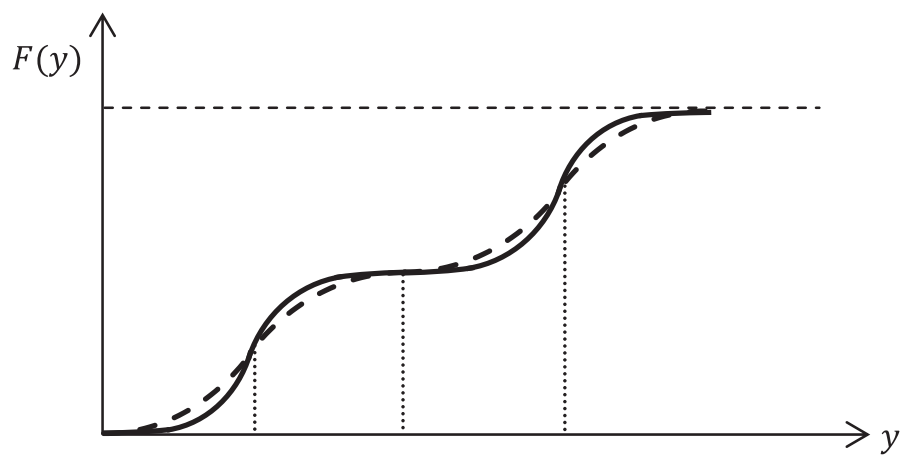

Abbildung 5: Verteilungsfunktionen mit mehreren Schnittpunkten

Die Ergebnisse lassen sich wie folgt zusammenfassen:

1. Bei einem konstanten Bonus oberhalb einer Gewinnschwelle ist es für den Manager allein massgeblich, mit welcher Wahrscheinlichkeit er diese Schwelle erreicht. Dies zeigt die Verteilungsfunktion an: Je niedriger der Funktionswert bei der Gewinnschwelle, desto grösser die Wahrscheinlichkeit, diese Schwelle zu erreichen. Es kommt also zu einem Risikoanreiz, wenn die Gewinnschwelle in einem Intervall liegt, in dem bei höherem Risiko die Verteilungsfunktion niedriger verläuft. Bei Verteilungen mit nur einem Schnittpunkt ergibt sich der Risikoanreiz daher bei Gewinnschwellen, die oberhalb des Schnittpunktquantils liegen, während es bei kleineren Gewinnschwellen nicht zum Risikoanreiz kommt. Bei mehreren Schnittpunkten gibt es jeweils mehrere solche Intervalle. Kommt es zu einem Risikoanreiz, ist dieser um so grösser, je höher die Bonuszahlung ausfällt.

2. Die Eigenkapitalnorm hat auf diesen Zusammenhang keinen Einfluss.

3. Sieht der Bonus eine proportionale Beteiligung an einer positiven Gewinngrösse vor (bilanzieller Gewinn oder Residualgewinn), entsteht in jedem Fall ein Risikoanreiz. Dieser ist um so grösser, je höher die Beteiligung am Gewinn ausfällt.

4. Der Einfluss der Eigenkapitalnorm auf die Risikowahl hängt zum einen davon ab, wie die Risikowahl die Wahrscheinlichkeit dafür beeinflusst, dass der Manager in den Bereich positiver Boni kommt, zum anderen davon, wie die Eigenkapitalnorm die Kapitalkosten (Fremdkapitalkosten beim bilanziellen Gewinn, gesamte Kapitalkosten beim Residualgewinn) beeinflusst. Die Argumentation zu Punkt 1. zeigt, dass für die genaue Ausformulierung der ersten Bedingung differenzierte Fallunterscheidungen vorzunehmen sind. Aus der zweiten Bedingung folgt aber unbedingt: Führt beim bilanziellen Gewinn als Bemessungsgrundlage für den Bonus die Verschärfung der Eigenkapitalnorm zu einer Minderung des Risikoanreizes, erhöht bei Verwendung des Residualgewinns als Bemessungsgrundlage mehr Eigenkapital den Risikoanreiz und umgekehrt.

Die schlechte Nachricht in Bezug auf die Robustheit der Ergebnisse des einfachen Basismodells ist, dass die spezifische Gestalt der Verteilungen viele Einzelergebnisse beeinflusst, so dass nur fallbezogene Aussagen getroffen werden können. Dagegen ist es die gute Nachricht, dass zwei Ergebnisse keiner Relativierung bedürfen: 1. Gibt es einen Risikoanreiz, so wird er durch einen höheren Bonus verstärkt. 2. Für jedwede Klasse von Verteilungen gibt es Bonussysteme, bei denen die Eigenkapitalnorm das Risikoanreizproblem nicht tangiert, es mildert oder es verschärft. Ohne zugleich die dem Manager vermittelten Anrei- 
ze zu untersuchen, lässt sich also die Wirkung einer Verschärfung der Eigenkapitalnorm nicht beurteilen.

Der Fokus des Manuskripts liegt auf der Interaktion zwischen Eigenkapitalnorm und Bonussystem bei der Bestimmung des in Banken übernommenen Risikos. Die Aussagen zu dieser Interaktion werden durch die vorgestellten Modifikationen des einfachen Basismodells nicht tangiert, sie sind also allgemeingültig.

\section{Diskussion}

In der mit Bedacht schlicht gehaltenen Untersuchung bleiben zwangsläufig einige Fragen offen. Die Implikationen zweier Aspekte sollen nun kurz abgeschätzt werden:

1. Welche weiteren Möglichkeiten zur Steuerung der Managerentscheidungen bestehen und was sagen bankaufsichtliche Vorschriften dazu? Offensichtlich wurden sowohl die Handlungsparameter des Managers als auch die Instrumente zu deren Steuerung nur in einer sehr elementaren Weise einbezogen. Aus der Analyse realer Bonussysteme ist beispielsweise bekannt, dass der Zusammenhang zwischen Bemessungsgrundlage und Bonus keineswegs proportional ist, sondern häufig den folgenden stilisierten Verlauf hat (vgl. Murphy/Jensen 2011, 3):

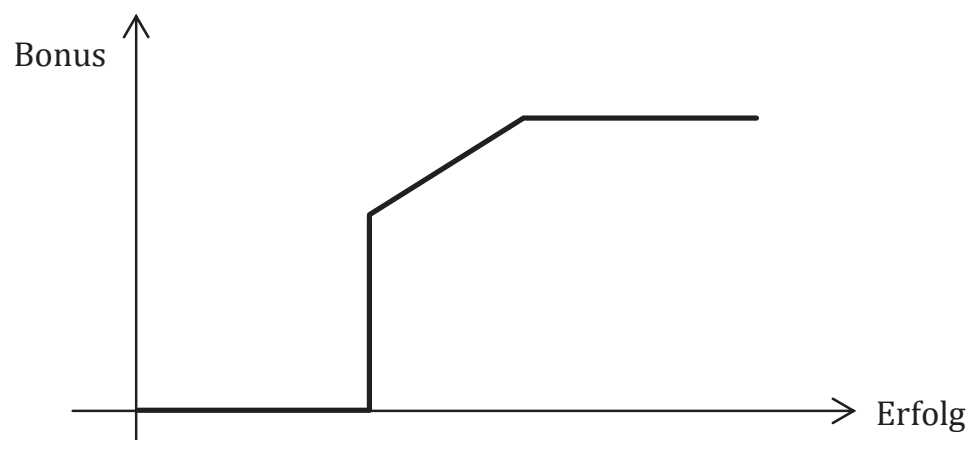

Abbildung 6: Stilisierter Verlauf der Bonus-Funktion

Der funktionale Zusammenhang zur Bemessungsgrundlage ist zunächst konvex, anschliessend aber konkav. Die Limitierung am oberen Ende steht einem extremen Risikoanreiz entgegen. Für die Begrenzung des Risikoanreizes ist auch die Möglichkeit eines negativen Bonus von Bedeutung; für dessen praktische Umsetzung ist auf das Modell einer BonusBank zu verweisen (ebenda, 14 f.). Das bedeutet, die Boni werden nur zum Teil unmittelbar ausbezahlt, zum Teil aber einbehalten und nur dann verzögert ausbezahlt, wenn der frühere Bonusanspruch nicht durch einen negativen Bonus infolge später realisierter Misserfolge aufgezehrt wird. Eine solche Regelung ist durch die deutsche Institutsvergütungsverordnung bereits heute bankaufsichtlich vorgegeben und wird laut CRD IV auch europaweit einzuführen sein. In der Sicht des vorgetragenen Modells bedeutet die Verrechnung von positiven Boni mit späteren negativen Boni eine Verminderung der Konvexität, so dass der Risikoanreiz abgeschwächt wird. Entsprechend weisen empirische Ergebnisse darauf hin, dass eine aufgeschobene Auszahlung von Boni das systemische Risiko von Banken verringert (Bosma/Koetter 2013). 
2. Die in den Bonusplänen herangezogenen Bemessungsgrundlagen werden hier etwas holzschnittartig dargestellt. Mit Blick auf den bilanziellen Gewinn sei daher darauf aufmerksam gemacht, dass dem Manager in Form der Ausnutzung von Ansatz- und Bewertungsspielräumen zusätzliche Handlungsspielräume zuwachsen, die ebenfalls Auswirkungen auf das Risiko und die Entlohnung haben. Mit Blick auf die unterschiedliche Wirkung verschiedener Bemessungsgrundlagen wird unter Verweis auf die Eigentümerinteressen für die Verwendung des Residualgewinns statt des bilanziellen Gewinns plädiert (Murphy/ Jensen 2011, 32 ff.), freilich mit ausdrücklichem Verweis auf ein drohendes Überinvestitionsproblem, wenn die Kapitalkosten nur teilweise einbezogen werden. Die Dimension abnehmender Skalenerträge der investierten Mittel wurde hier nicht einbezogen, so dass sich auch insoweit nochmals differenzierte Resultate ergeben könnten.

Für die Beurteilung dieser offenen Punkte ist festzustellen, dass sich die Hauptaussage des Beitrags gerade auf das im Fehlen eindeutiger Zusammenhänge bezieht: Der Einfluss der Eigenkapitalnorm kann eine gegebene Konvexität verstärken, abschwächen oder überhaupt nicht tangieren. Die Übertragbarkeit der hier in Abschnitt 3 angerissenen Robustheitsprüfung ist intuitiv leicht einzusehen: Wenn schon bei einer sehr einfachen Modellierung uneindeutige Ergebnisse resultieren, kann keinesfalls erwartet werden, dass in einer allgemeineren Modellierung das Ergebnis eindeutig ausfällt.

Abschliessend sei daher noch einmal auf die Erkenntnis hingewiesen, dass es bei einer krisenvorbeugenden Bankenaufsicht weniger darauf ankommt, den Einsatz einzelner Instrumente zu perfektionieren, als darauf, ein ausgewogenes und Interaktionen berücksichtigendes Paket von Regelungen in Kraft zu setzen (so auch Laeven/Levine 2009). Angesichts der elementaren Wirkung einer stärkeren Internalisierung externer Effekte werden strenge Eigenkapitalnormen ganz gewiss zu diesem Paket gehören. Vor dem Hintergrund der hier aufgezeigten Situationsabhängigkeit der Wirkung verschiedener Anreizsysteme ist es eine anzustrebende Folge strenger Eigenkapitalnormen, dass sie die Eigentümer davon abhalten, über die Gestaltung von Anreizsysteme die Manager zu mehr Risiko zu provozieren. Weitere hilfreiche Beiträge zur Internalisierung bestehen in einer Einlagensicherung, die einen vollen Risikoausgleich über Prämien vorsieht, und in Vorkehrungen, die jedweden Bail-out ausschließen. Damit sind zugleich zwei der drei zentralen Bausteine der Europäischen Bankenunion umschrieben (vgl. bspw. Krabnen 2013, 168 f.).

\section{Literaturhinweise}

Admati, A.R./De Marzo, P.M./Hellwig, M.F./Pfleiderer, P. (2011): Fallacies, Irrelevant Facts, and Myths in the Discussion of Capital Regulation: Why Bank Equity is Not Expensive, Stanford GSB Research Paper No. 2063, March 2011.

Bai, G./Elyasiani, E. (2013): Bank Stability and Managerial Compensation, in: Journal of Banking and Finance, Jg. 37, S. 799-813.

Bannier, C.E./Feess, E./Packham, N. (2013): Competition, Bonuses, and Risk-taking in the Banking Industry, in: Review of Finance, Jg. 17, S. 653-690.

Bebchuk, L.A./Cohen, A./Spamann, H. (2010): The Wages od Failure: Executive Compensation at Bear Stearns and Lehman 2000-2008, ECGI Finance Working Paper No. 287, June 2010.

Bhagat, S./Bolton, B. (2013): Misaligned Bank Executive Incentive Compensation. Mimeo, June 2013. 
Bhagat, S./Bolton, B./Lu, J. (2012): Size, Leverage, and Risk-taking of Financial Institutions, August 2012, Electronic copy available at: http://ssrn.com/abstract=2122727.

Bosma, J.J./Koetter, M. (2013): Bank Executive Compensation and Systemic Risk, Paper Presented at the EEA-ESEM 2013, February 2013.

Burghof, H.-P./Rudolph, B. (1996): Bankenaufsicht. Theorie und Praxis der Regulierung, Wiesbaden.

Chaigneau, P. (2012): Risk-shifting and the Regulation of Bank CEO's Compensation, in: Journal of Financial Stability, Jg. 9, S. 778-789. http://dx.doi.org/10.1016/j.jfs.2012.08.003.

Fablenbrach, R./Stulz, R.M. (2011): Bank CEO Incentives and the Credit Crises, in: Journal of Financial Economics, Jg. 99, S. 11-26.

Gabaix, X./Landier, A. (2008): Why Has CEO Pay Increased So Much?, in: Quarterly Journal of Economics, Jg. 123, S. 49-100.

Hakenes, H./Schnabel, I. (2010): Banks without Parachutes: Competitive Effects of Government Bail-out Policies, in: Journal of Financial Stability, Jg. 6, S. 156-168.

Hakenes, H./Schnabel, I. (2013): Bank Bonuses and Bail-outs, Preprints of the Max Planck Institute for Research on Collective Goods 2013/3, February 2013.

Krahnen, J.P. (2013): Rettung durch Regulierung? Eckpunkte des Liikanen-Berichts, in: Perspektiven der Wirtschaftspolitik, Jg. 14, S. 167-185.

Laeven, L./Levine, R. (2009): Bank Governance, Regulation and Risk Taking, in: Journal of Financial Economics, Jg. 93, S. 259-275.

Luz, G./Neus, W./Schaber, M./Schneider, P./Wagner, C.-P./Weber, M. (Hrsg.) (2013): CRR visuell. Die neuen EU-Vorschriften der Capital Requirements Regulation, Stuttgart.

Mang, E./Albrecht, B. (2011): Struktur und Höhe der Vorstandsvergütung: Fakten und Mythen, in: Zeitschrift für betriebswirtschaftliche Forschung, Jg. 63, S. 858-881.

Merton, R.C. (1974): On the Pricing of Corporate Debt: The Risk Structure of Interest Rates, in: Journal of Finance, Jg. 29, S. 449-470.

Murphy, K.J./Jensen, M.C. (2011): CEO Bonus Plans: And How to Fix Them, September 2011, Electronic copy available at: http://ssrn.com/abstract=1935654.

Neus, W. (2013): Einführung in die Betriebswirtschaftslehre, 8. Aufl., Tübingen.

Rothschild, M./Stiglitz, J.E. (1970): Increasing Risk: I. A Definition, in: Journal of Economic Theory, Jg. 2, S. 225-243.

Schaber, M. (2000): Bankenaufsicht und Kapitalkonsolidierung. Eine Konfrontation handelsrechtlicher Normen mit bankaufsichtlichen Erfordernissen, Wiesbaden.

Vallascas, F./Hagendorff, J. (2012): CEO Remuneration and Bank Default Risk: Evidence from the U.S. and Europe, January 2012, Electronic copy available at: http://ssrn.com/abstract=1691754.

Neus Werner, Prof. Dr. ist Inhaber des Lehrstuhls für Bankwirtschaft an der EberhardKarls-Universität Tübingen.

Anschrift: Eberhard-Karls-Universität Tübingen, Abteilung Bankwirtschaft, Nauklerstr. 47, D-72074 Tübingen, Tel.: +49 (0)7071/29-72575, Fax: +49 (0)7071/29-5882, E-Mail: werner.neus@uni-tuebingen.de 